\title{
O comportamento do recém-nascido internado na uti neonatal quando exposto aos sons intrauterinos
}

\section{Behavior of the newborn intern in the neonatal icu when exposed to intrauterine sounds}

DOI: $10.46814 /$ lajdv3n3-016

Recebimento dos originais: 01/05/2021

Aceitação para publicação: 30/06/2021

\section{Paula G. Cachambú}

Graduanda do Curso de Graduação em Enfermagem da Universidade Regional Integrada do Alto Uruguai e das Missões URI-Erechim.

\section{Irany Achiles Denti}

Irany Achiles Denti. Doutor em Ciências da Saúde pela UNESC-SC. Professor do Curso de Graduação em Enfermagem da URI - Erechim

\section{Luana Ferrão}

Enfermeira. Mestre em Envelhecimento humano pela UPF - Professora do Curso de Graduação em

Enfermagem da URI - Erechim.

\section{Cibele Sandri Manfredini}

Enfermeira. Doutora em Saúde da Criança pela PUC - RS. Professora do Curso de Graduação em Enfermagem da Universidade Regional Integrada do Alto Uruguai e das Missões URI- Erechim. Endereço: Rua Pedro Alvarez Cabral 492/801- Centro - Erechim RS.

E-mail: cibelem@uricer.edu.br

\section{RESUMO}

O útero materno permite ao feto repouso e sono profundo, sendo que imerso nesta estrutura, o feto já registra algumas experiências, as quais permitem que tenham lembranças deste período após o nascimento. O objetivo do estudo foi descrever o comportamento do recém-nascido, internado Unidade de Terapia Intensiva Neonatal (UTIN), quando exposto ao som uterino. Trata-se de um estudo quaseexperimental, com 7 Recém-Nascidos (RNs) de 29 a 38 semanas, internados nesta unidade, no período de setembro a outubro de 2019. Os participantes foram expostos ao som uterino por 15 minutos ininterruptos dentro da incubadora, com Leq abaixo de 60,0 dBA. Como elementos do estudo foram avaliadas a Frequência Respiratória, Frequência Cardíaca, Saturação de Oxigênio. Também foi utilizado a escala da dor no recém-nascido e no lactente - Neonatal Infant Pain Scale (NIPS), além da avaliação dos estados de sono e vigília adaptada de Brazelton, antes e após a exposição do som uterino. Resultados: Identificou-se que após a exposição ao som uterino os RNs apresentaram redução da Frequência Respiratória (FR) e Frequência Cardíaca (FC). A escala da dor apresentou significância estatística $\mathrm{p}<0,05$ e a avaliação dos estados de sono e vigília apresentou significância estatística de $\mathrm{p}<0,01$ a $\mathrm{p}<0,05$ após a exposição do som uterino. Conclusão: a exposição do som uterino mostrou eficácia para mitigar o controle da dor e melhorou os períodos da vigília e do sono dos RNs.

Palavras-chave: Neonato. Medição da Dor. Neonatologia 


\section{ABSTRACT}

The maternal uterus allows the fetus rest and deep sleep, still into the uterus, the fetus already has some experiences, the ones that allow them to have memories from the uterine life after born. General objective knowing the newborn behaviour, hospitalized UTIN, when exposed to the uterine sound. It has an almost- experimental study, with 7 new-borns from 29 to 38 weeks, hospitalized in the UTIN, September to October 2019. The participants were exposed to the uterine sound. It was verified the Breath Frequency, Hearth frequency, Oxygen Saturation, applied pain scale in the new-born and in the infant (NIPS) and evaluation of the sleep stages and the adapted vigil of Brazelton, before and after the uterine sound exposition. Results: it was identified that after the uterine sound exposition the new borns showed reduction of BF and HF. The pain scale showed significant statistics from $\mathrm{p} 0.05$ after the sound exposition, and the evaluation of the sleep stages and adapted vigil, showed significant statistics of $\mathrm{p}$ 0,01 o p 0,05 after uterine sound exposition. Conclusion: with this study it was possible to identify that the application of the uterine sound can be used as technique to help in the reduction of the pain in the new-born and keep them calmer and quieter.

Key words: New Born. Pain counting. Neonatology

\section{INTRODUÇÃO}

A Unidade de Terapia Intensiva Neonatal (UTIN) é um local onde ficam pacientes graves ou parcialmente graves, os quais necessitam de monitorização contínua e cuidados complexos. Devido à variedade de equipamentos de suporte à vida existentes neste local, como monitores, ventiladores mecânicos, incubadoras, bombas de infusão e a grande circulação de pessoas, ele tornou-se um ambiente ruidoso (CARDOSO et al., 2015).

A internação do RN na UTIN ocorre em situações de prematuridade, instabilidade clínica e necessidade de monitorização contínua, com equipamentos que são específicos desta unidade (SPOSITO, 2016). Em função disto torna-se um ambiente estressante principalmente pela exposição à luz intensa, ruídos excessivos e procedimentos invasivos ou não. Isso pode contribuir para que o RN apresente estresse, alteração no ciclo do sono e dor (JORDÃO et al., 2016).

O recém-nascido é mais susceptível a dor do que as crianças e os adultos, já que as vias anatômicas, neurofisiológicas e hormonais estão prontas ao nascimento, e as vias capazes de inibir ou reduzir a dor ainda não estão formados (NAZARETH et al., 2015). Ao ser internado na UTIN ele é submetido a procedimentos invasivos e dolorosos, cuidados de rotina e manuseio excessivo pelos profissionais da saúde inclusive os da enfermagem, totalizando de 130 a 234 manipulações em 24 horas, muitas delas dolorosas (ROCHA; MARTINS, 2017).

Com a permanência na UTIN o RN pode apresentar diversas alterações, tais como: alteração do sistema circulatório, respiratório, comportamental, imunológico, dentre outros. Podendo ainda ocasionar consequências em longo prazo, comprometendo o desenvolvimento e crescimento do RN (RODRIGUES; SOUZA; WERNWCK, 2016). 
Por vários anos acreditava-se que o RN era um ser incapaz de sentir dor, devido seu sistema nervoso não estar totalmente formado. Com o passar dos anos, foi se observando que as vias anatômicas responsáveis pela dor começavam a ser desenvolvidas a partir da sétima semana de gestação e entorno da vigésima semana eram distribuídas em toda superfície corporal. Com 24 semanas de gestação, os estímulos dolorosos estão associados com marcadores fisiológicos, metabólicos e hormonais de resposta ao estresse. Com 30 semanas, a mielinização das vias nociceptivas está completa (NAZARETH et al., 2015).

O útero materno permite ao feto repouso e sono profundo, sendo o meio ideal para que ocorra o processo de crescimento e maturação das estruturas anatômicas, fisiológicas e neurofisiológicas, relativas ao desenvolvimento fetal. A UTIN proporciona aos neonatos um ambiente bem diferente do mundo intrauterino. A assistência necessária o expõe a manuseios constantes, à dor e a outras formas de estimulação de natureza sensorial, tais como forte luminosidade, ruídos provocados por movimentos humanos e pelo manuseio de materiais. O neonato sofre, assim, a interrupção dos seus ciclos de sono, o que pode contribuir para o surgimento do estresse, dificultando o processo de desenvolvimento orgânico (PINTO et al., 2008).

O comportamento do RN pode ser avaliado através da escala da dor no recém-nascido e no lactente (Neonatal Infant Pain Scale-NIPS). Esta escala se baseia nas alterações comportamentais do $\mathrm{RN}$, sendo composta por cinco indicadores comportamentais de dor: expressão facial, choro, movimentação de braços e pernas, estado de consciência e um indicador fisiológico que é a respiração (NAZARETH et al., 2015)

O Neonato, completamente adaptado ao meio intrauterino, onde não tem luminosidade, onde sofre uma contenção física em meio líquido e uma estimulação auditiva e vestibular provocada pelos movimentos maternos, ao nascer, encontra no novo ambiente excesso de luminosidade, ruídos, movimentos constantes e interrupções dos seus ciclos de sono (FREITAS et al, 2018). O Recémnascido está muito mais susceptível às influências do meio extrauterino por não possuir ainda maturação fisiológica suficiente, o que poderá acarretar inúmeras complicações. Apesar do avanço tecnológico com novos equipamentos e terapêuticas, o útero continua sendo o local ideal para o desenvolvimento psico-bio-fisio-neuromotor do feto (FREITAS et al., 2018).

Por anos o RN era tido como uma substância amorfa e pensava-se que somente após o nascimento, ele começaria a exercer suas funções. Apenas há algumas décadas constatou-se que ainda dentro do útero, ele já tem algumas experiências, as quais permitem que tenham lembranças da vida uterina após o nascimento. O feto pode ouvir diversos sons vindos do corpo da mãe, tais como: batimentos cardíacos, movimentos peristálticos, respiração, fluxo do cordão umbilical, dentre outros, que juntos produzem o som intrauterino característico. Os batimentos cardíacos da mãe é a primeira 
experiência rítmica experimentada pelo feto e em segundo é a vibração do cordão umbilical (JABER, 2013).

A exposição a sons intrauterinos pode acalmar RN que estão nervosos ou chorando. A técnica dos sons intrauterinos, como método terapêutico para acalmar os neonatos, vem sendo estudada a partir da década de 70. (BURTON e DOHERTY, 1979). No entanto, após este período, existe uma lacuna com ausência de estudos atuais sobre o assunto.

A exposição de RNs agitados aos sons intrauterinos é capaz de acalmar os mesmos. Um estudo realizado em 1975 com 87 recém-nascidos apontou que 73 deles, acalmaram-se após a reprodução dos sons intrauterinos (MUROOKA, 1975). Em outro estudo foram expostos aos sons maternos, quatorze bebês prematuros entre 26 e 32 semanas de gestação, com o objetivo de examinar os efeitos dos sons biológicos maternos sobre a estabilidade cardiorrespiratória (apnéias, bradicardias) em bebês prematuros. O estudo demonstrou, em curto prazo, a capacidade de regulação cardiorrespiratória dos prematuros durante a exposição dos sons maternos, uma vez que os bebês são capazes de reconhecer esses sons e responder para o ambiente auditivo, aumentando assim a capacidade de regulação cardiorrespiratória (DOHENY et al., 2012).

Durante as atividades laborais e acadêmicas na UTIN, foi possível observar que os RNs têm diversas reações aos sons que são expostos, por necessidade dos equipamentos para manutenção da vida. Após leituras sobre o tema, surgiu o questionamento: Qual seria o comportamento do recémnascido, internado na UTIN, quando exposto ao som uterino? A partir disso, realizou-se este estudo com o objetivo geral de conhecer o comportamento do recém-nascido, internado na UTIN quando exposto ao som uterino, e objetivos específicos verificar o nível da dor do RN, antes e após a exposição ao som uterino; avaliar frequência cardíaca, frequência respiratória, saturação de oxigênio e comparar o comportamento de RNs antes e após a exposição ao som intrauterino; descrever as características dos recém-nascidos expostos ao som uterino.

\section{METODOLOGIA}

Trata-se de um estudo quase-experimental realizado em uma Unidade de Terapia Intensiva Neonatal de um Hospital Geral do Norte do Rio Grande do Sul, com RNs, internados nesta unidade. Foi utilizado como critérios de inclusão, recém-nascidos com idade gestacional igual ou maior que 28 semanas, segundo Arnon (2011) é o período ideal para se inicie a terapia musical, e que pelo menos um responsável legal tenha autorizado a participação no estudo. Como critérios de exclusão, recémnascidos que estavam sobre efeito de medicações para sedação; em ventilação mecânica; estado clínico grave com restrição de ruídos; alguma situação clinica não prevista, que no momento da coleta foi observada pela equipe da unidade e que impediu a participação no estudo. Este estudo respeitou a 
Resolução 466/2012 que dispõe sobre estudos envolvendo seres humanos, sendo aprovado pelo Comitê de Ética em Pesquisa sob parecer no 3.527.510.

Mediante as devidas autorizações, a enfermeira responsável pela unidade indicou os possíveis participantes da pesquisa. No momento em que seus responsáveis legais estavam presentes na UTIN foi realizado a primeira abordagem, expondo os objetivos, metodologia e questões éticas.

Além dos dados dos prontuários foi realizado a avaliação da frequência cardíaca, frequência respiratória, saturação periférica de oxigênio, aplicação da escala da dor no recém-nascido e no lactente- NIPS e escala de avaliação dos estados de sono e vigília adaptada de Brazelton. Em seguida, os participantes do estudo foram expostos ao som intrauterino conforme Rodrigues et al, (2018) por um período de 15 minutos ininterruptos dentro da incubadora, por meio de caixas de som já instaladas pela instituição, seguindo os preceitos da Associação Brasileira de Normas Técnicas, a qual estabelece valores de Leq abaixo de 60,0 dBA, e para o cumprimento deste quesito, foi utilizado o decibelímetro para aferição e manutenção dos decibéis durante todo o período de aplicação. $\mathrm{O}$ som aplicado foi o mesmo utilizado por Murooka (1974) em seus estudos. Após a exposição ao som, foi avaliado novamente frequência cardíaca, frequência respiratória, saturação periférica de oxigênio, escala da dor e escala de avaliação dos estados de sono e vigília adaptada de Brazelton. Este procedimento foi aplicado em três momentos, sendo um no turno da manhã, um da tarde e um da noite.

A Escala da dor no recém-nascido e no lactente (Neonatal Infante Pain Scale-NIPS) avalia o comportamento do recém-nascido baseada em indicadores de dor. A escala avalia cinco parâmetros comportamentais e um indicador fisiológico: expressão facial, choro, movimentação corporal (braços e pernas), estado de alerta e respiração (MATSUDA et al., 2014). A pontuação da escala pode variar de 0 a 7. A expressão facial, respiração, estado de alerta e movimentação de braços e pernas têm pontuação de 0 a 1 e o choro pontuação de 0 a 2 (LAWRENCE et al., 1993; PEREIRA et al., 1999). A pontuação varia de 0 a 7 , sendo que valor de 3 alguma conduta já deverá ser tomada, e pontuação igual ou superior a 4 é definido a presença de dor (ALMEIDA; PEDUTTI, 2018).

A avaliação do comportamento do RN utilizando-se a escala de Brazelton, foi baseada na linguagem que o mesmo apresentou em relação aos estados de sono e vigília. A escala avalia seis estados comportamentais: sono profundo; sono ativo; sonolência; alerta tranquilo; despertar ativo e choro intenso, recebendo pontuação de 1 a 6 respectivamente (SANTOS, 2016).

O tratamento dos dados foi efetuado através de estatística descritiva e a significância estatística $(\mathrm{p}<0,05)$ foi definida pelo teste Post Hoc de Tukey. Os resultados estão apresentados em tabela e figuras. 


\section{RESULTADOS}

No primeiro momento da coleta de dados, ocorreu o registro das características de cada participante através de informações do prontuário. Estes estão representados na Tabela I e são referentes ao sexo, idade gestacional, motivo da internação, peso de nascimento e peso no dia da aplicação do som de cada RN. Para caracterizar os participantes foi definida a sigla RN com um número de 1 a 7 na sequência, respeitando o anonimato.

Tabela I- Características dos recém-nascidos

\begin{tabular}{|c|c|c|c|c|c|c|}
\hline & SEXO & IG & PESO NASC & PESO ATUAL & IDADE ATUAL & $\begin{array}{l}\text { MOTIVO DE } \\
\text { INTERNAÇÃO }\end{array}$ \\
\hline RN 1 & $\bar{F}$ & $32 \mathrm{~S}$ & $1.532 \mathrm{gr}$ & $1.940 \mathrm{gr}$ & 24 DIAS & PREMATURIDADE \\
\hline RN 2 & M & $29 \mathrm{~S}$ & $1.324 \mathrm{gr}$ & $1.324 \mathrm{gr}$ & 31 DIAS & PREMATURIDADE \\
\hline RN 3 & M & $29 \mathrm{~S}$ & $1.180 \mathrm{gr}$ & $2.212 \mathrm{gr}$ & 31 DIAS & PREMATURIDADE \\
\hline RN 4 & $\mathrm{M}$ & $35 \mathrm{~S}$ & $2.066 \mathrm{gr}$ & $2.232 \mathrm{gr}$ & 13 DIAS & PREMATURIDADE \\
\hline RN 5 & M & $37 \mathrm{~S}$ & $1.904 \mathrm{gr}$ & $1.842 \mathrm{gr}$ & 14 DIAS & BAIXO PESO \\
\hline RN 6 & M & $35 \mathrm{~S}$ & $2.398 \mathrm{gr}$ & $2.374 \mathrm{gr}$ & 06 DIAS & PREMATURIDADE \\
\hline RN 7 & M & $38 \mathrm{~S}$ & $3.800 \mathrm{gr}$ & $3.670 \mathrm{gr}$ & 06 DIAS & $\begin{array}{l}\text { HIPERTERMIA } \\
\text { GEMÊNCIA }\end{array}$ \\
\hline
\end{tabular}

Fonte: o estudo.

Participaram do estudo sete recém-nascidos que estiveram internados na Unidade de Terapia Intensiva Neonatal sendo seis $(86 \%)$ do sexo masculino e um $(14 \%)$ do sexo feminino. A idade gestacional (IG) no momento de nascimento variou entre 29 e 38 semanas, sendo que $29 \%$ tinha 29semanas e $71 \%$ deles tinham IG entre 32 e 38 semanas de gestação.

$\mathrm{O}$ peso de nascimento variou de $1.180 \mathrm{gr}$ a $3.800 \mathrm{gr}$, sendo que $57 \%$ dos RN tiveram o peso abaixo de $2 \mathrm{KG}$ e $43 \%$ entre $2 \mathrm{KG}$ e 3.800 gr. O peso no momento da coleta de dados já havia alterado, ficando entre $1.324 \mathrm{gr}$ e $3.670 \mathrm{gr}$, sendo $57 \%$ dos RN com peso acima de $2 \mathrm{KG}$ e $43 \%$ com peso abaixo de $2 \mathrm{KG}$. Esta alteração do peso configura-se pelo fato da coleta de dados ter ocorrido quando os RNs já tinham entre seis dias e trinta e um dias de vida. Dois dos $\mathrm{RN}$ foram considerados a termo, um foi internado na UTIN em função de uma Hipertermia com gemência e o outro por baixo peso, os outros cinco foram internados na UTIN em função da prematuridade.

Os dados coletados através da avaliação e observação dos recém-nascidos antes e após a exposição ao som uterino estão representados separadamente em cinco figuras. Sendo que cada uma apresenta os resultados do turno da manhã, tarde e noite. 
Figura I - Efeito da aplicação do som uterino sobre a frequência cardíaca (FC) de recém-nascidos internado na UTIN. (A) frequência cardíaca antes e depois da exposição ao som, no turno da manhã; (B) frequência cardíaca antes e depois da exposição ao som, no turno da tarde; (C) frequência cardíaca antes e depois da exposição ao som no turno da noite. A significância estatística foi definida pelo teste Post Hoc de Tukey de múltiplas comparações.

\section{FREQUÊNCIA CARDÍACA ANTES E DEPOIS DA EXPOSIÇÃO AO SOM}

A

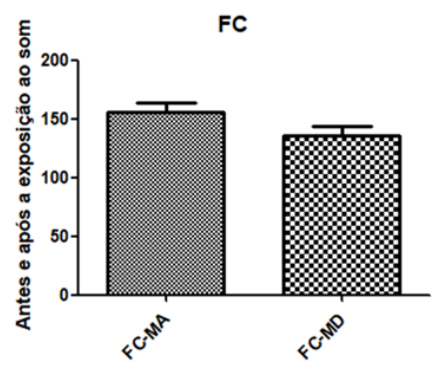

B

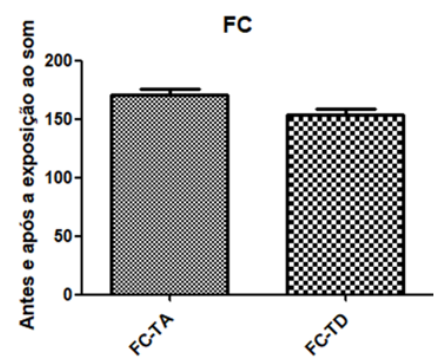

C

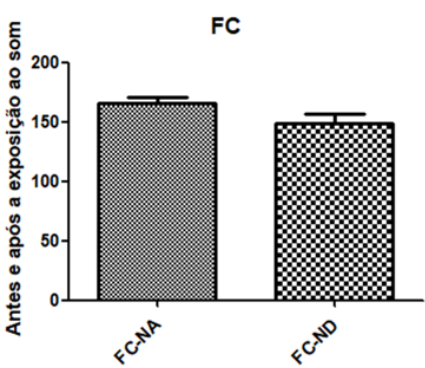

A frequência cardíaca foi avaliada antes e depois da exposição ao som uterino no turno da manhã, tarde e noite. A figura I mostra diminuição da frequência cardíaca após a exposição ao som uterino, sendo mais acentuada no turno da manhã, no entanto não apresentou significância estatística. Antes da exposição, no turno da manhã, a frequência cardíaca variou de 120bpm a 182bpm e após a exposição foi de $112 \mathrm{bpm}$ a $160 \mathrm{bpm}$. Mesmo não tendo significância estatística é possível dizer houve resposta clinica positiva e que a aplicação do som uterino colaborou com a estabilidade ou redução da frequência cardíaca neste grupo de recém-nascidos.

Figura II - Efeito da aplicação do som uterino sobre a Frequência Respiratória (FR) de recém-nascidos internado na UTIN. (A) frequência respiratória antes e depois da exposição ao som, no turno da manhã; (B) frequência respiratória antes e depois da exposição ao som, no turno da tarde; (C) frequência respiratória antes e depois da exposição ao som no turno da noite. A significância estatística foi definida pelo teste Post Hoc de Tukey de múltiplas comparações.

\section{FREQUENCIA RESPIRATÓRIA ANTES E DEPOIS DA EXPOSIÇÃO AO SOM}

A

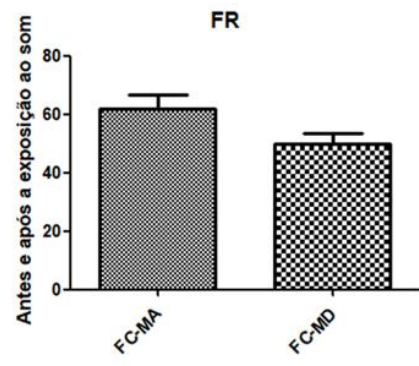

B

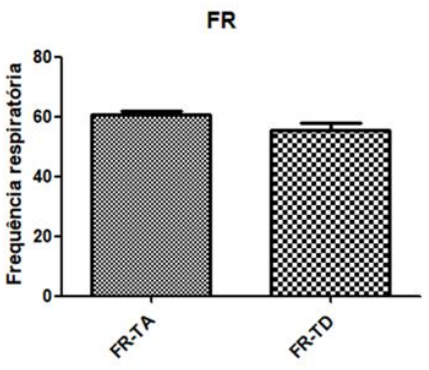

C

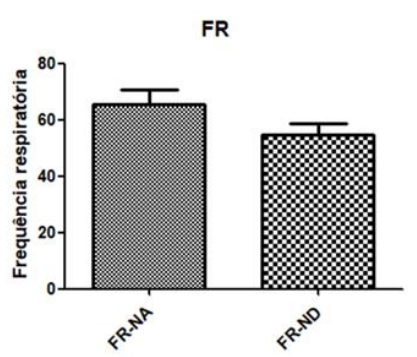

Em relação à Frequência Respiratória é possível visualizar na figura II que no turno da tarde não apresentou alteração antes e depois da exposição ao som. Sendo que no turno da manhã e da noite 
após a exposição ao som uterino, visualiza-se diminuição da frequência respiratória, não tendo significância estatística. No turno da manhã a FR antes da exposição variou de $40 \mathrm{mpm}$ a $75 \mathrm{mpm}$ e após a exposição variou de $38 \mathrm{mpm}$ a $62 \mathrm{mpm}$. No turno da noite a FR antes da exposição ficou entre $45 \mathrm{mpm}$ a $84 \mathrm{mpm}$ e após a exposição variou de $40 \mathrm{mpm}$ a $64 \mathrm{mpm}$. Com as variações, apresentadas por este grupo de recém-nascidos, da frequência respiratória antes e após a exposição ao som uterino, é possível inferir que esta técnica pode ser útil para estabilizar da frequência respiratória, sendo este um importante marcador clínico.

Figura III - Efeito da aplicação do som uterino sobre a Saturação de Oxigênio $\left(\right.$ Sat. $\left._{2}\right)$ de recém-nascidos internado na UTIN. (A) saturação de Oxigênio antes e depois da exposição ao som, no turno da manhã; (B) saturação de Oxigênio antes e depois da exposição ao som, no turno da tarde; (C) saturação de Oxigênio antes e depois da exposição ao som no turno da noite. A significância estatística foi definida pelo teste Post Hoc de Tukey de múltiplas comparações.

\section{SATURAÇÃO DE OXIGÊNIO ANTES E DEPOIS DA EXPOSIÇÃO AO SOM}

\section{A}

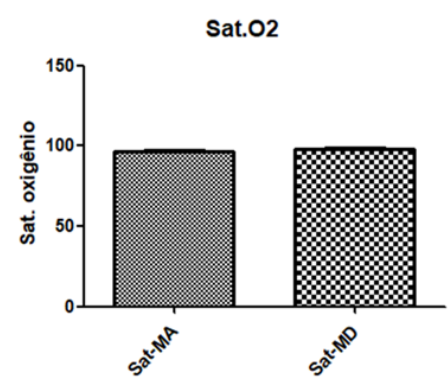

B

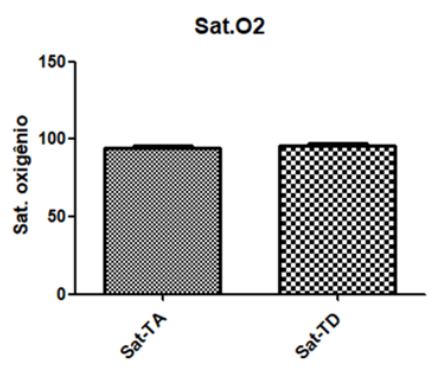

C

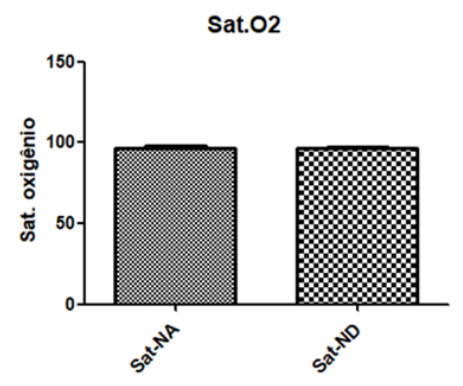

A aplicação do som uterino não apresentou efeito sobre a saturação de oxigenio, nos recémnascidos internados na UTIN, podendo ser visualizdo na figura III que nos três turnos, manhã, tarde e noite, antes e após a exposição a saturação de oxigênio se manteve nos mesmos parâmetros. Apresentou uma variação entre $95 \%$ a $100 \%$ nos turnos da manhã e noite e no truno da tarde a variação foi entre $88 \%$ a $100 \%$. Não apresentando significancia estatistica. 
Figura IV - Efeito da aplicação do som uterino sobre a Dor, com a utilização da Escala de NIPS, em recém-nascidos internado na UTIN. (A) dor antes e depois da exposição ao som, no turno da manhã; (B) dor antes e depois da exposição ao som, no turno da tarde; (C) dor antes e depois da exposição ao som no turno da noite. A significância estatística foi definida por *p<0,05, pelo teste Post Hoc de Tukey de múltiplas comparações.

\section{ESCALA DA DOR (NIPS) ANTES E DEPOIS \\ DA EXPOSIÇÃO AO SOM}

A

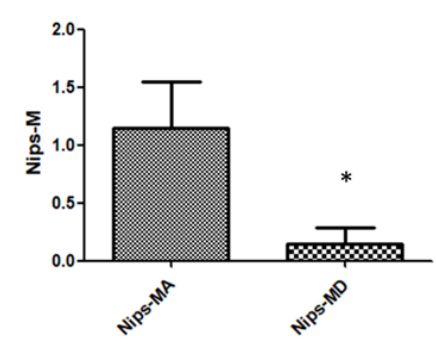

B

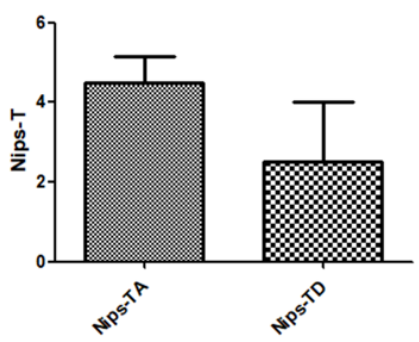

C

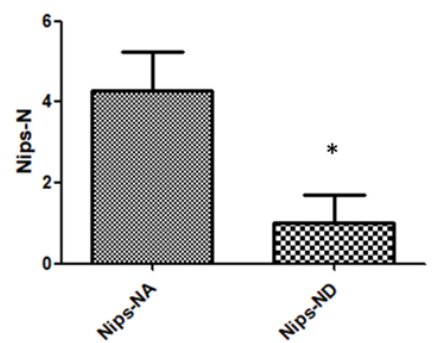

Neste estudo observou-se alterações significativas na escala da dor antes e após a exposição ao som uterino nos três turnos avaliados. No turno da manhã ocorreu a maior alteração, apresentando significância estatística de $\mathrm{p}<0,05$, sendo os valores anterior a exposição ficando entre 0 e 3 e após a exposição de 0 a 1 . Sendo que o RN que apresentou a pontuação 1 após a exposição se manteve estável, pois antes da exposição, também presentou a pontuação 1 .

A avaliação da dor no turno da noite apresentou significância estatística de p<0,05, apresentando uma variação de parâmetros antes e depois que ficou respectivamente, antes da exposição de 0 a 7 e depois da exposição de 0 a 5. Observa-se que os RNs que apresentaram pontuação 7 antes do som uterino, foram avaliados e passaram a ter pontuação de 1 a 0 . Isto demonstra que a utilização do som uterino teve efeito sobre a dor neste grupo de recém-nascidos.

Figura V - Efeito da aplicação do som uterino sobre os estados de sono e vigília, com a utilização da Escala de Brazelton, em recém-nascido internado na UTIN. (A) comportamento antes e depois da exposição ao som, no turno da manhã; (B) comportamento antes e depois da exposição ao som, no turno da tarde; (C) comportamento antes e depois da exposição ao som no turno da noite. A significância estatística foi definida por $* \mathrm{p}<0,05$ e $*^{*} \mathrm{p}<0,01$ pelo teste Post Hoc de Tukey de múltiplas comparações.

ESCALA DE AVALIAÇÃO DOS ESTADOS DE SONO E VIGÍLIA DE BRAZELTON ANTES E DEPOIS DA EXPOSIÇÃO AO SOM

A

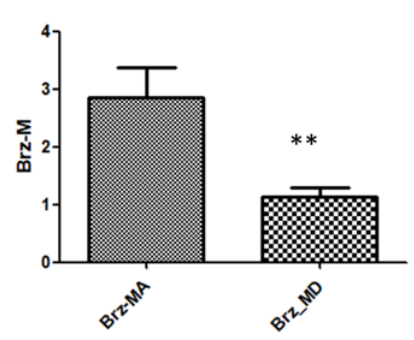

B

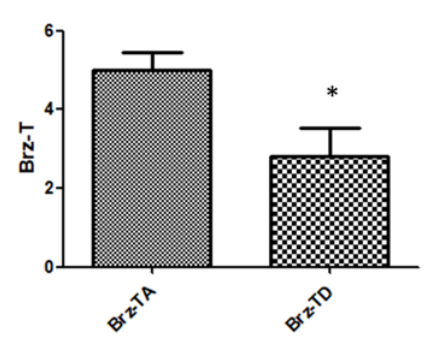

C

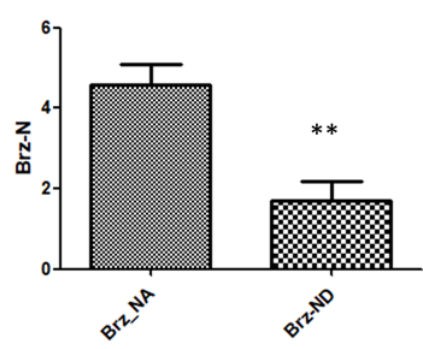


A figura $\mathrm{V}$ refere-se ao efeito da utilização do som uterino no comportamento do $\mathrm{RN}$ antes e após a exposição ao som. A Fig. V(A) apresenta a pontuação da escala do turno da manhã antes e após a exposição ao som, com significância estatística de $p<0,01$. Neste turno a pontuação variou de 1 a 4 antes da exposição e após a pontuação foi 1, tendo apenas um RN com pontuação 2, sendo que antes da exposição este RN teve a pontuação 4.

A fig. V(B) mostra o comportamento antes e após a exposição ao som uterino para o turno da tarde, teve significância estatística de $\mathrm{p}<0,05$. Onde a variação da pontuação antes da exposição foi de 4 a 6 e depois da exposição ficou entre 1 e 5, sendo que o RN que teve pontuação 5 depois, teve antes da exposição pontuação 6 .

A Fig. V (C) mostra que pela análise estatística apontou significância estatística de $p<0,01$ para o comportamento do RN antes e após a exposição ao som uterino no turno da noite. Antes da exposição o comportamento variou, na escala, de 2 a 6 e após a exposição a variação foi de 1 a 4 . O RN que apresentou a pontuação 4 depois da exposição teve pontuação 5 antes da exposição, mostrando que todos os RN tiveram redução na pontuação após o som.

\section{DISCUSSÃO}

A prematuridade é um problema que afeta uma parcela significativa da população de crianças recém-nascidas, acarretando problemas devidos ao déficit de várias funções neurológicas. Além das perdas devidas ao crescimento e desenvolvimento, estes problemas determinam aumento dos custos com as internações hospitalares assim como para as famílias que necessitam de aporte financeiro e logística para atender a uma criança que em vários aspectos necessita de cuidados especiais após a alta hospitalar.

As condições fisiológicas do nascimento prematuro e, por conseguinte, a imaturidade de vários aparelhos e sistemas, pode ser determinada por vários problemas atribuídos à gestação assim como do comportamento materno incluindo o uso de drogas e a alimentação. No estudo atual, pela sua natureza e objetivos, todas as crianças eram prematuras e com baixo peso (mostrado na tabela I). Efeitos específicos e similares para a prole devidos à dieta foram encontrados por (SEONG et al., 2019). Neste estudo os pesquisadores descrevem que a ingestão materna de dieta com a adição de frutose em ratos aumentou a pressão arterial das genitoras e da prole além de ocasionar distúrbios metabólicos. Distúrbios metabólicos, redução no número e peso de fetos de ratas tratadas com frutose também foram relatados por (ZIN, et al., (2021). Muito embora estas referências abordem dados de pesquisa com animais, possivelmente estes possam ser correlacionados com o comportamento dietético de gestantes e destacam a importância dos cuidados com a gestação para possivelmente ocorrer redução na prematuridade em seres humanos. Na sequência serão discutidos os achados pertinentes aos benefícios 
da exposição das crianças prematuras a sons biológicos gerados principalmente pela passagem do sangue pelos vasos adjacentes ao útero.

A exposição aos sons maternos biológicos, traz benefícios para o sistema cardiorrespiratório dos recém-nascidos, podendo, portanto, ser cruciais para o desenvolvimento da saúde do RN. Os bebês são capazes de reconhecer esses sons e responder ao ambiente auditivo mais reconfortante, aumentando assim a capacidade de regulação cardiorrespiratória (DOHENY et al., 2012).

Análoga a exposição aos sons uterinos, segundo Oliveira (2014) a intervenção da música na UTIN pode trazer benefícios para os recém-nascidos, pois reduz o comportamento de estresse e melhora a regulação da frequência cardíaca. O que corrobora com o atual estudo onde foi possível identificar redução dos parâmetros da frequência cardíaca após a exposição do som materno.

Estudo realizado por Portugal et al. (2017) com recém-nascidos prematuros, mostrou que quando estes são estimulados aos sons maternos apresentam maior capacidade fisiológica para autorregularão da respiração, mantendo-a estável, o que reduz a probabilidade de apneia ou hipóxia. Este vem ao encontro dos dados encontrados no atual estudo onde foi possível identificar redução dos parâmetros da frequência respiratória após a exposição do som materno, no turno da manhã e da noite. DOHENY et al. (2012) referem que os sons maternos podem ser importantes principalmente na ausência da mãe por fornecer ambiente relaxante e melhorar a capacidade de autorregulação de funções vitais como a respiração e a frequência cardíaca.

Apesar do presente estudo não ter apresentado mudanças significativas na saturação periférico de oxigênio nos recém-nascidos avaliados, Portugal et al. (2017) e Oliveira et al. (2014) citam que a estimulação auditiva utilizando técnicas de sons, tem efeito extremamente benéfico, embora os valores da oximetria periférica estejam dentro dos limites normais. Afirmam ainda a importância de se considerar que o nível de oxigênio se mantenha dentro dos padrões fisiológicos, a fim de reduzir o número de sequelas.

A equipe de enfermagem deve ser capaz de identificar, avaliar e tratar a dor do recém-nascido, para que os danos ou prejuízos decorrentes da internação do RN na UTIN sejam minimizados. Existem alguns instrumentos para avaliar a dor do recém-nascido, sendo a escala da dor no recém-nascido e no lactente (Neonatal Infante Pain Scale-NIPS) uma delas. A partir dos resultados da aplicação da escala, os profissionais definem condutas farmacológicas e não farmacológicas para prevenir e minimizar a dor, as quais tornam os cuidados de enfermagem mais humanizados (CORREIA, 2011).

Diversos tipos de musicoterapia, vem sendo utilizada como métodos não farmacológicos e alternativos para acalmar os recém-nascidos, sendo que existem efeitos benéficos na estabilização dos dados vitais, no estado de sono/vigília, na redução de estresse, ganho de peso e no desenvolvimento psicológico e cognitivo de RNs (RODRIGUES et al., 2018). 
As medidas não farmacológicas são eficazes, quando utilizadas na prevenção e controle da dor de leve intensidade. Essas medidas são estratégias que tem como objetivos, principalmente, prevenir a intensificação da dor, o estresse e a agitação, bem como minimizar as complicações da mesma. A musicoterapia pode ser implementada como um método não farmacológico, pois promove distração e desvio da atenção dolorosa para algo agradável. A música provoca relaxamento, reduzindo a dor, estimulando a liberação de endorfina e diminuindo da mesma forma o estresse (CORREIA, 2011). Seguindo esta linha de pensamento, o estudo atual mostrou que a exposição ao som materno o RN apresentou diminuição da dor e que este pode ser utilizado como uma medida não farmacológica para minimizar a dor no RN internado na UTIN.

O estudo com métodos não farmacológicos realizado por Tandoi et al., (2014) para o controle do estresse pós-natal de prematuros, diz que a música melhora o estado de comportamento do RN, que a exposição aos sons maternos é crucial para o desenvolvimento saudável do RN, e que, tem efeito calmante, capaz e reduzir o efeito de estresse adaptativo resultante do nascimento.

Estudo realizado por Burton e Doherty (1979), com a reprodução dos sons uterinos, foi verificado que estes têm a capacidade de acalmar os bebês que estão agitados ou chorando, a atividade realizada foi avaliada através da escala de Brazelton, antes e durante a exposição aos sons. Os resultados obtidos indicam que os sons uterinos acalmam $90 \%$ dos bebês que estão nervosos ou chorando e que não tem efeitos evidentes em bebês que apenas estão em estado de alerta. O estudo atual demonstrou que o comportamento do RN após ser exposto ao som uterino sofreu alteração, no sentido do mesmo ter ficado mais calmo e tranquilo. Além disto observou-se que os RN que estavam dormindo ou que estavam chorosos em função do horário próximo a alimentação, a aplicação do som uterino não teve influência no comportamento.

Após o nascimento, o bebê tem lembranças de sons ouvidos durante sua vida uterina, principalmente de sons provenientes da mãe, fazendo com que após o nascimento, recorde destes sons e, que fique mais atento principalmente a sons humanos do que a não humanos. Os bebês, ao ouvir sons, com periodicidade, semelhantes aos batimentos cardíacos humano, acalmam-se com maior facilidade do que quando expostos a outros sons (NUNES, 2009).

\section{CONCLUSÕES}

O trabalho evidenciou clinicamente que os sons intrauterinos podem ser utilizados como ferramenta para acalmar os recém-nascidos. Após a exposição ocorreu melhorias nos parâmetros de FC e FR; já no que diz respeito à saturação de oxigênio, não houve alteração significativa. Com relação 
à escala da dor (NIPS) e na avaliação dos estados de sono e vigília, os dados estatísticos apontaram significância $\mathrm{p}<0,05$ e $\mathrm{p}<0,01$ quando comparados antes e após a exposição ao som intrauterino.

O estudo aponta potenciais benefícios para a aplicação do som, como uma técnica não invasiva para complementar os cuidados atuais realizados em unidades de terapia intensiva neonatal, sendo possível tornar o ambiente mais agradável e acolhedor, proporcionando o atendimento mais humano.

Como limitações da pesquisa aponta-se a amostra reduzida, em função de que no período da coleta de dados não houve muita rotatividade de pacientes na UTIN, pois estes têm perfil de longa permanência na unidade. Além disso identificou-se um reduzido número de estudos realizados com essa temática.

Como sugestão seriam necessários mais estudos com número amostral mais amplo, no que se refere à aplicação dos sons uterinos para acalmar os recém-nascidos. 


\section{REFERÊNCIAS}

ARNON, S. Music therapy interventionin the neonatal intensive care unit environment. Jornal de Pediatria, Rio de Janeiro, v. 87, n. 3, p. 183-185, 2011.

ALMEIDA, R. P; PEDUTTI, S. [org]. Linha de cuidado da criança: manual de neonatologia, $2^{\mathrm{a}}$ ed. São Paulo: SES/SP, 2018.

BURTON, S. R; DOHERTY. The Response of Neonates to Intra-uterine. Develop. Med. Child Neurol, v. 21, P. 723-729, 1979.

CARDOSO, S. M. S; KOZLOWSKI, L. C; LACERDA, A. B. M; MARQUES, J. M; RIBAS, A. Respostas fisiológicas de neonatos frente a ruídos em unidade neonatal. Brazilian Journal Otorhinolaryngology, São Paulo, v. 81, n. 6, p. 583-588, Nov-Dez, 2015.

CORREIA, C. I. M. Fatores que influenciam os enfermeiros na adoção de estratégias não farmacológicas para o controlo da dor do recém-nascido. ESCOLA SUPERIOR DE ENFERMAGEM DO PORTO, Dissertação de Mestrado, 2011.

DOHENY, L; HURWITZ, S; INSOFT, R; RINGER. S; LAHAV. A. Exposure to biological maternal sounds improves cardiorespiratory regulation in extremely preterm infants. The Journal of MaternalFetal and Neonatal Medicine, EUA, v. 25, n. 9, p. 1591-1594, 2012.

FREITAS, M. C. N; SOUSA, A. O. B; CABRAL, S. A. A. O; ALENCAR, M. C. B; GUEDES, M. S. S. G; OLIVEIRA, G. F. Caracterização dos Recém-nascidos Internados em Unidades de Terapia Intensiva. Id online Revista Multidisciplinar e de Psicologia, v. 12, n 40, p. 228-242, 2018. Disponível em: https:<//idonline.emnuvens.com.br/id/article/view/1110/1619> Acesso em: 22 Abril de 2018.

JABER, M. S. O bebê e a música: sobre a percepção e a estruturação do estímulo musical, do pré-natal ao segundo ano de vida pós-natal. Dissertação (mestrado) em música. Universidade Federal do Rio de Janeiro, Faculdade de Música, Rio de Janeiro, 2013.

JORDÃO, K. R; PINTO, L. A. P; MACHADO, L. R; COSTAS, L. B. V. L; TRAJANO, E. T. L. Possíveis fatores estressantes na unidade de terapia intensiva neonatal em hospital universitário. Revista Brasileira de Terapia Intensiva, v. 28, n. 3, p. 310-314, 2016.

LAWRENCE, J; ALCOCK, D; MCGRATH, P; MACMURRAY, S. B; DULBERG. C. The development of a tool to assess neonatal pain. Neonatal Network, n. 12, v. 6, p. 59-66, 1993.

MATSUDA, M. R; MARTINS, M. R; FILHO, M. A. N; MATTA, A. C. G. Métodos não farmacológicos no alivio da dor no recém-nascido. Brazilian Journal of Surgery and Clinical Research, n. 5, v. 1, pg. 59-63, Dez 2013- Fev 2014.

Miracle Drug: Dr. Hajime Murooka's "Lullaby from the Womb" LP

Disponível em: $<$ https://www.youtube.com/watch?time_continue=2\&v=BGGm9-csV-I $>$ Acesso em: 10 de Maio de 2019.

MUROOKA, H. Lullaby from the womb, New York: Cpitol Records, 1974. 
MURROKA. Sleep Gently in Womb. Tokyo: Toshiba EMI Ltd, 1975.

NAZARETH, C. D; LAVOR, M. F. H; SOUSA, T. M. A. S. Ocorrência de dor em bebês internados em unidade de terapia intensiva neonatal de maternidade terciária. Revista de Medicina da UFC, Ceará, v. 55, n. 1, p. 33-37, 2015.

NUNES, P. A. O. Experiência auditiva no meio intrauterino. Psicologia.pt- O portal dos psicólogos, 2009.

OLIVEIRA, M. F; OSELAME, G. B; NEVES, E. B; OLIVEIRA, E. M. Musicoterapia como ferramenta terapêutica no setor da saúde: Uma revisão sistemática. Revista da Universidade Vale do Rio Verde, Três Corações, v. 12, n. 2, p. 871-878, Ago-Dez, 2014.

PINTO, E. F; SILVA, I. L; CARDOSO, F. BERESFORD, H. O estresse no neonato pré-termo: uma reflexão axiológica acerca de possíveis influências dos fatores sensório-ambientais em unidades de terapia intensiva neonatal. Fit Perf J, Rio de Janeiro, v. 7, n. 5, p. 345-51, Set-Out, 2008.

PORTUGUAL, C. M. G. S. L; SÁ, L.O; AREIAS, M. H. F. G. P. Cardiorespiratory effects of maternal sounds in infants born between 26 and 33 weeks of gestation. Revista de Enfermagem Referência, Coimbra, Série IV, n. 12, p. 65-64, Jan-Fev, 2017.

RODRIGUES, D. I. S; FÓFANO, G. A; BARREIROS, L. L; COUTO, C. S. F; VIEIRA, C. F; OLIVEIRA, M. A. A. C. A utilização da musicoterapia na assistência ao prematuro internado em unidade de terapia intensiva neonatal: uma revisão bibliográfica. Revista Científica Fagoc Saúde, v. 3, n. 1, p. 67-73, 2018.

RODRIGUES, J. R; SOUSA, D. S; WERNECK. A. L. Identificação e avaliação da percepção dos profissionais de enfermagem em relação a dor/desconforto do recém-nascido. Arq. Ciênc. Saúde, São José do Rio Preto, v. 23, n. 1. p. 27-31, Jan-Mar, 2016.

ROCHA, L. A; MARTINS, C. D. Ruídos ambientais na uti neonatal: considerações da equipe de enfermagem. Revista Brasileira de Ciências da Vida, v. 5, n.4. p. 1-23, Dez, 2017.

SANTOS, A. M. G. Repercussões do posicionamento corporal no estado fisiológico e comportamental de recém-nascido pré-termo. Universidade estadual do oeste do paraná, Cascavel, 2016.

SEONG, H.O.; CHO, H.M.; KIM, M.; KIM, I. Maternal High-Fructose Intake Induces Multigenerational Activation of the Renin-Angiotensin-Aldosterone System. Hypertension. v.74, n. 3, p.00-00, 2019.

SPOSITO, N. P. B. Prevalência e manejo da dor em recém-nascidos internados em unidade de terapia intensiva neonatal: estudo longitudinal. Dissertação (mestrado). Escola de Enfermagem da Universidade de São Paulo, São Paulo, 2016.

TANDOI, F; FRANCESCATO, G; PAGANI. A; BUZZETTI. G; NEGRI, E; AGOSTI, M. "The Original Sound": a new non-pharmacological approach to the postnatal stress management of preterm infants. The Jounal of Maternal-Fetal e Neonatal Medicine, v. 28, n. 16, pg. 1934-1938, Nov, 2014.

ZIN, L; TONELO, A. ; ROMAN, S.S.; CICHOTA, L.C.; AMAFRAL. A.U.; DENTI, I.A. influência da frutosemia crônica sobre parâmetros de crescimento e desenvolvimento, maternos, fetais, 
bioquímicos e estresse oxidativo de ratas wistar. Latin American Journal of Development, Curitiba, v. 3, n. 2, p. 819-838, mar./apr. 2021. 